\title{
Safety margin against the fatigue fracture under random loading
}

\author{
Martin Garan ${ }^{1}$, Vladimir Chmelko ${ }^{2 *}$, and Ervin Schafer $^{1}$ \\ ${ }^{1}$ Slovak University of Technology, Faculty of Mechanical Engineering, Department of Applied Mechanics and Mechatronics, Námestie \\ Slobody 17, 81231 Bratislava, Slovakia \\ ${ }^{2}$ Faculty of Mechanical Engineering, Regional Technological Institute, University of West Bohemia, Univerzitní 22, 30614 Plzeň, \\ Czech republic
}

\begin{abstract}
The fatigue lifetime of the structure in operation is express in the number of working cycles, kilometers or time depending units. By harmonic process of loading is possible to express the safety margin as reserve against the required lifetime in the form of number cycles or amplitude of loading. By nonharmonic character of loading process in operation is difficult to express the level of safety margin as the reserve of loading (safety margin in loading). On the case of towing trailer will be discussed more ways of expression the reserve (safety margin) of structure against the fatigue fracture for required fatigue lifetime. For experimental acquired loading process in the most loaded cross-section will be discussed concept of determination of the level safety as reserve in the form of loading parameters. In the case, when the peaks of loading process exceeding the material yield stress (in the most loaded point of the structure) will be discussed concept of expression of loading in the form of time-varying process of strain. By using the cyclic material properties obtained in mode with controlled total strain is possible the safety margin to express also as the multiple of process loading parameters.
\end{abstract}

\section{Concept of fatigue lifetime assessment}

We will use the "safe life" approach to assess the fatigue life of a real construction. This approach is schematically illustrated in Fig. 1

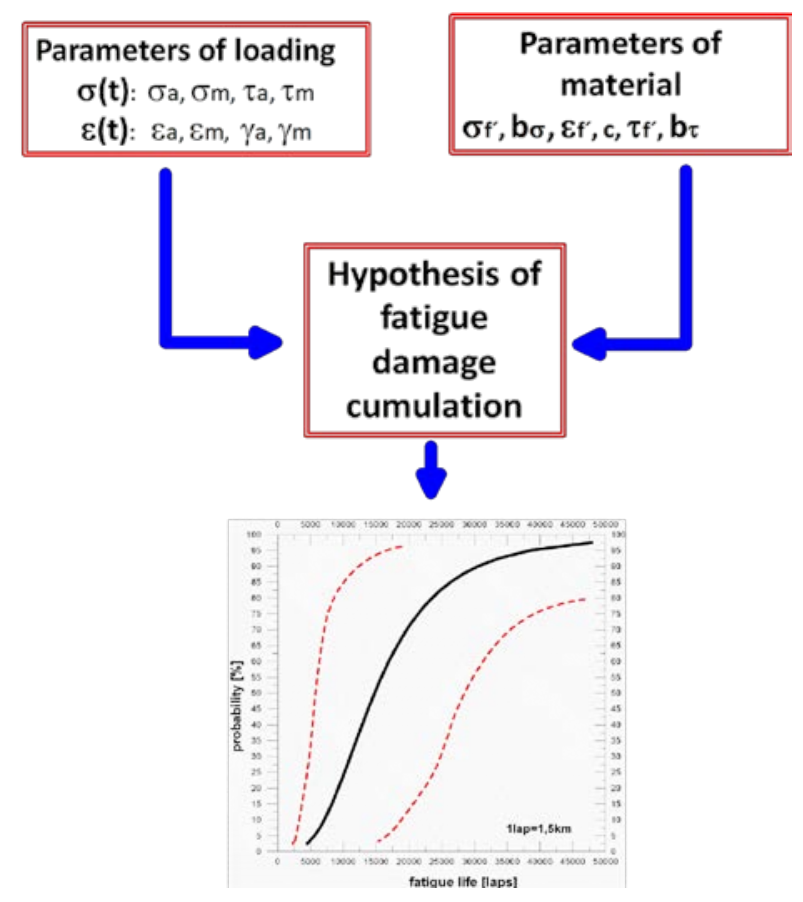

Fig. 1 The concept of fatigue lifetime assessment
Load input parameters are the time deformation curves respectively. tension in the form of individual components. The most credible way to get them is to measure them directly during real-time construction. Before performing the measurement, it is necessary to identify the critical cross-sections i.e. critical points of the structure. They are the places with the highest variable stress component, which determines the minimum fatigue life of the structure as a whole. At the critical point of the construction (one or more), it is then necessary to obtain the desired time-varying processes of the stress of the representative length and content by direct measurement. The second set of inputs required are the cyclic properties of the material that are required by the chosen criterion of fatigue damage cumulation. Due to the scattering of the cyclic properties of the material as well as the variation of the individual realizations of the loading process, it is necessary to use the statistic approach for estimating the fatigue lifetime developed in [1] and practically used e.g. in [2]. As a result of such an approach for fatigue lifetime evaluation (including scattering of individual input parameters), is then the fatigue lifetime distribution function, which may take account of the randomness of the occurrence of the material parameters or of the load parameters or of the two groups of parameters. In the Fig. 2 is an example of a fatigue lifetime distribution function, where for required fatigue lifetime probability of survival (e.g., $95 \%$ ) is marked the guaranteed value of the fatigue lifetime of the critical place of assessed structure.

* Corresponding author: vladimir.chmelko@stuba.sk 


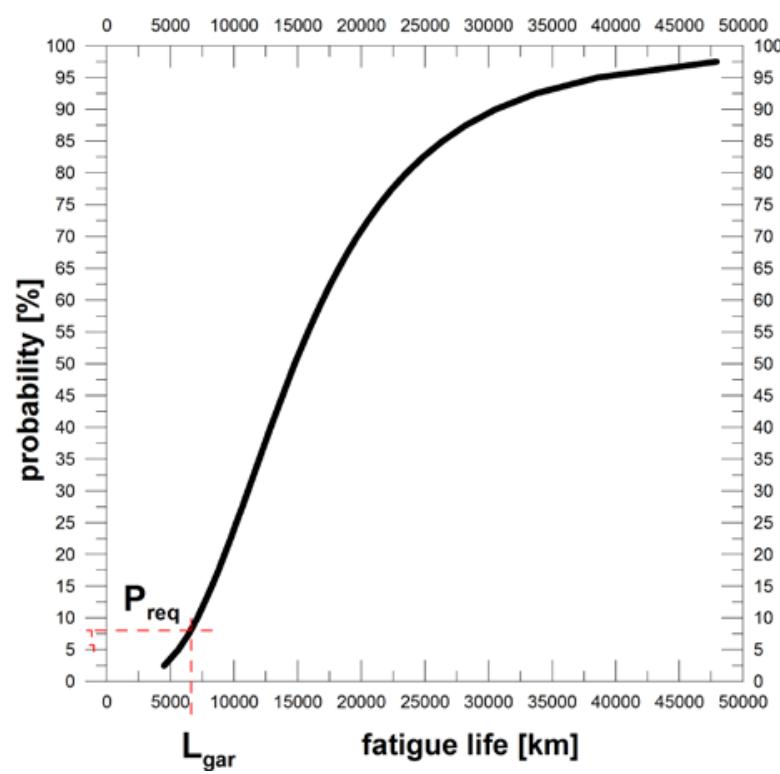

Fig. 2 The guaranteed fatigue lifetime for the required probability of survival

\section{The measurement of loading parameters}

The subject of assessment is the axle that serves as the chassis of a caravan. It consists of a main beam of cross section $82 \times 4 \mathrm{~mm}$ from the steel St52, at the ends of which are the arms with the wheel units. On both sides at a distance of $180 \mathrm{~mm}$ from the wheel's axis, a trapezoidal bracket is attached to the beam to fix the spring bars. The borders on both sides of the console serve to correctly attach the axle to the chassis and drive the caravan. To fix the mutual position of the axle with the caravan undercarriage, there are 4 screw connections on each of the brackets. As the axles used in caravans have to be braked, the center of the beam is a rack and cantilever beam for rear brake. (Fig .3).

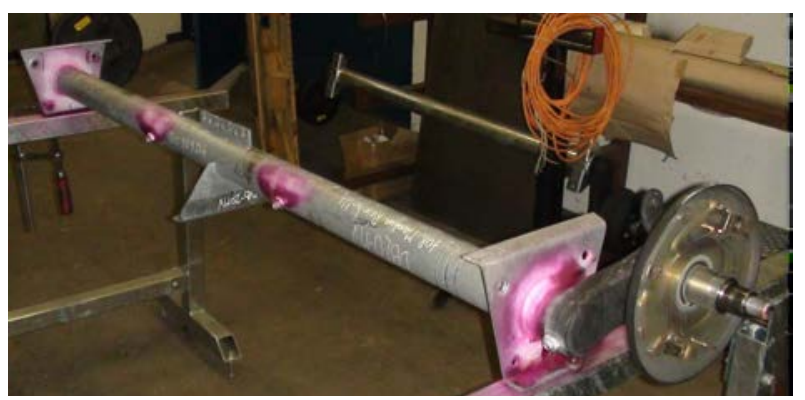

Fig. 3. The assessed axle

The axle loading diagram is shown in Fig. 4. The most stressed cross-sections are the area between the points of attachment of the spring bars, where a combination of vertical bending and caravan weight and torque from the spring bars occurs. Less stress is the strain from side forces (tilting), vertical forces (tens) and bending from pulling force. The strain gauges were distributed around the center of the axle at the marked cross-section. The layout and strain gauge connection was chosen as shown in (Fig. 5) to determine the individual stresses in the cross-section and to find the point with the highest stress values $[3,4]$.

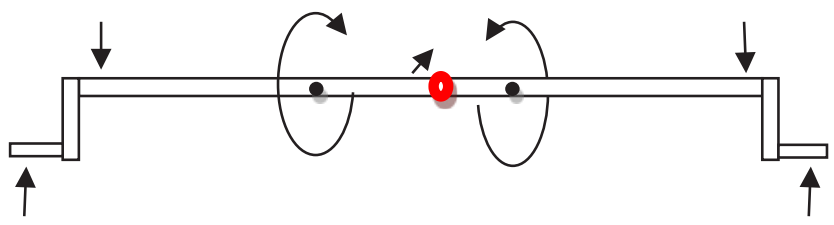

Fig. 4 Axle loading diagram. Black points indicate the location of the anchor bars and the red circle indicates the position of the sensor location

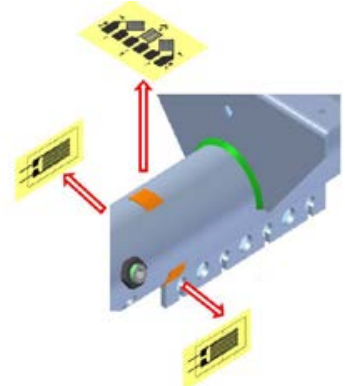

Fig. 5 Strain gauges arrangement around cross section

The stress measurement was performed during the test at the testing polygon. The typical stresses in the most stressed cross-sectional area of the measured crosssection for each type of road and two total load weights are shown in Fig. 6. Diagrams of the dominant stresses (vertical bending and torsion) are time-depending naturally and acting proportionally in phase.
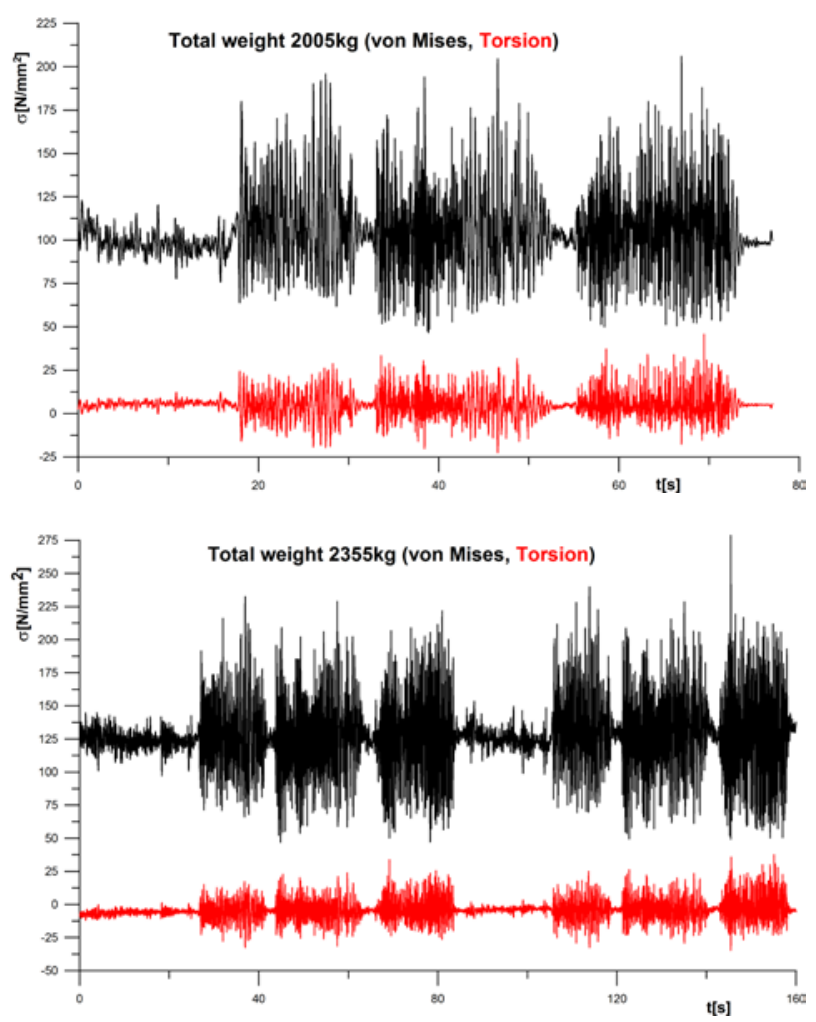

Fig. 6 Typical stresses at the most stressed cross-section point during driving on different road reliefs 


\section{The assessment of the fatigue lifetime safety by multiple of loading}

A sufficiently long loading record allowed a relevant assessment of fatigue lifetime at the most stressed point of axle. The calculation procedure considering the variance of the cyclic properties of material (St52) of the axle is obvious from the following scheme displayed in Fig.7.

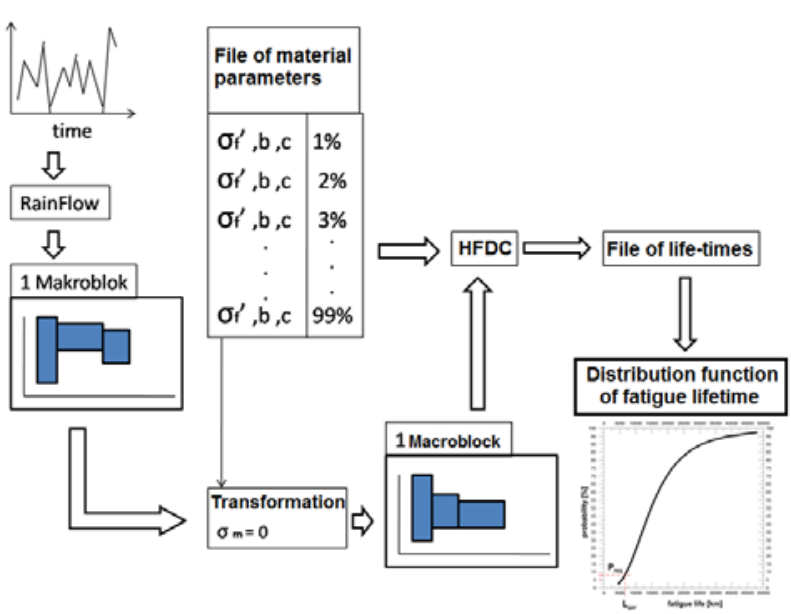

Fig. 7 The scheme of fatigue lifetime assessment with considering the variance of the material parameters

As criterion of the fatigue damage cumulation was used the energetic criterion by Kliman [4] in the form

$$
D=\frac{1}{N_{f M i n}} \sum n_{i}\left(\frac{\sigma_{a i}}{\sigma_{a M a x}}\right)^{\frac{b+c}{b}}
$$

where $\sigma_{a i}$ is an individual stress amplitude

$n_{i}$ is frequency of its occurrence

$\sigma_{\text {amax }}$ is the highest stress amplitude in the loading block

$N_{f m i n}$ is the number of cycles to the fracture corresponding to the highest stress amplitude

Fatigue lifetimes in kilometres of traffic operation for individual road reliefs and for selected probabilities of occurrence of material parameters (confidence limits, prediction limits) are displayed in Tab.1.

Tab. 1 Fatigue lifetimes for individual road reliefs (axle of a caravan)

\begin{tabular}{|c|c|c|c|}
\hline \multicolumn{2}{|c|}{ Asphalt road } & \multicolumn{2}{c|}{ Belgium paving } \\
\hline \multicolumn{2}{|c|}{ 2005kg [10 $\mathbf{k m ]}$} & \multicolumn{2}{c|}{ 2005kg [10 $\mathbf{1 0}^{\mathbf{k m}]}$} \\
\hline CI 95\% & 4123072274 & CI 95\% & 114.5 \\
\hline CI 5\% & 125199630 & CI 5\% & 3.17 \\
\hline PI 95\% & 8961754744.5 & PI 95\% & 313.8 \\
\hline PI 5\% & 76277760 & PI 5\% & 1.67 \\
\hline Reg.line & 933444075.6 & Reg.line & 27 \\
\hline
\end{tabular}

\begin{tabular}{|c|c|c|c|}
\hline \multicolumn{2}{|c|}{$2350 \mathrm{~kg}\left[10^{6} \mathrm{~km}\right]$} & \multicolumn{2}{|c|}{$2350 \mathrm{~kg}\left[10^{6} \mathrm{~km}\right]$} \\
\hline CI $95 \%$ & 4941080.6 & CI $95 \%$ & 1.7 \\
\hline CI $5 \%$ & 91402.1 & CI $5 \%$ & 0.0275 \\
\hline PI 95\% & 12391753.8 & PI 95\% & 5.2 \\
\hline PI $5 \%$ & 53486.2 & PI $5 \%$ & 0.014 \\
\hline Reg.čiara & 970959.1 & Reg.čiara & 0.34 \\
\hline \multicolumn{2}{|c|}{ Patch asphalt } & \multicolumn{2}{|c|}{ Potholes } \\
\hline \multicolumn{2}{|c|}{$2005 \mathrm{~kg}\left[10^{6} \mathrm{~km}\right]$} & \multicolumn{2}{|c|}{$2005 \mathrm{~kg}\left[10^{4} \mathrm{~km}\right]$} \\
\hline CI 95\% & 207.9 & CI 95\% & 6885.3 \\
\hline CI $5 \%$ & 6.6 & CI $5 \%$ & 242.2 \\
\hline PI 95\% & 565.4 & PI 95\% & 18638 \\
\hline PI 5\% & 3.4 & PI 5\% & 1265 \\
\hline Reg.line & 51.9 & Reg.line & 1787.6 \\
\hline \multicolumn{2}{|c|}{$2350 \mathrm{~kg}\left[10^{6} \mathrm{~km}\right]$} & \multicolumn{2}{|c|}{$2350 \mathrm{~kg}\left[10^{4} \mathrm{~km}\right]$} \\
\hline CI 95\% & 4.4 & CI $95 \%$ & 11.4 \\
\hline CI $5 \%$ & 0.089 & CI $5 \%$ & 0.17 \\
\hline PI 95\% & 13.17 & PI 95\% & 37.4 \\
\hline PI $5 \%$ & 0.046 & PI $5 \%$ & 0.0843 \\
\hline Reg.line & 0.95 & Reg.line & 2.27 \\
\hline
\end{tabular}

The fatigue lifetime values are calculated for two axle loadings $-2000 \mathrm{~kg}$ and $2350 \mathrm{~kg}$. From the measured stresses, it can be seen the increase of the peaks during increasing the loading and also shortening the calculated fatigue lifetime. A small (rarely) disputation is to express the degree of safety against fatigue fracture for operating loadings with time-depending stress diagrams. The degree of construction safety for this type of operation can be expressed in several ways:

- as a multiple of the required number of kilometers (i.e. time of operation) against the guaranteed one for the required risk of premature fracture (Fig.8)

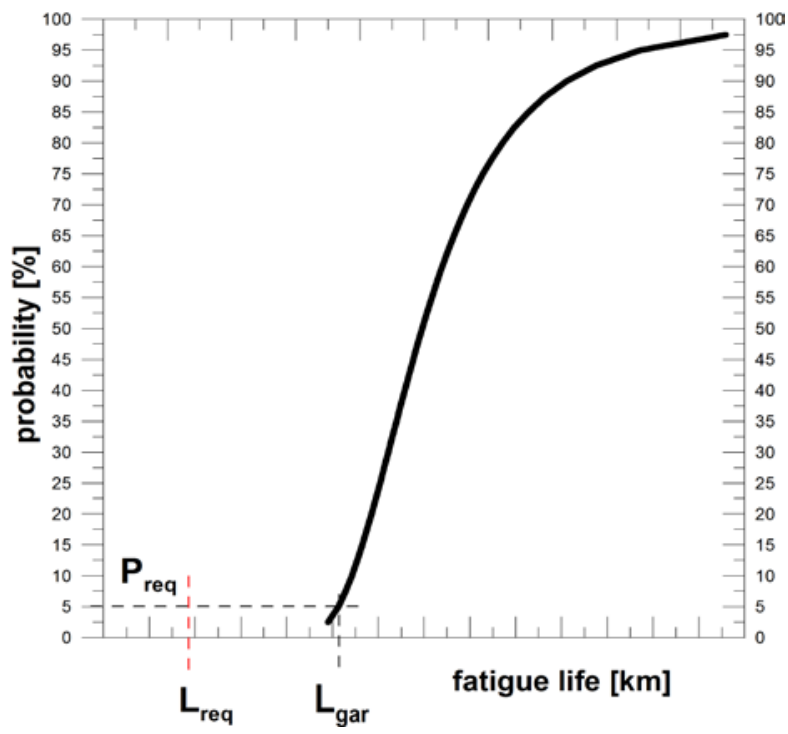

Fig. 8 The expression of the safety level $\mathrm{L}_{\text {guaranteed }} / \mathrm{L}_{\text {required }}$ at the time of operation for the permissible risk of premature fracture 
- as multiple the loading to which the structure was designed

- ...

There is no direct cause between the peak points at the critical point of the structure and the weight of the load (e.g. in the case of the assessed axle). Increased loading weight increases the mean value of the cycle and can also increases the cycle amplitudes as dynamic components of the loading process. The multiple of these increases is likely different. A slight increase in loading parameters could be, for example, multiplicity of the standard deviation of the loading process as the parameter of the dynamic loading component. Determining the value of such a multiple (loading safety) is not, however, possible directly. However, it is possible to create additional processes from the original loading process by multiplying its peaks by selected multiples. For each such obtained loading process, to calculate the fatigue lifetime according to the scheme in Fig. 7 and progress of the fatigue lifetime as the multiple of standard deviation display graphically. From obtained dependence with suitable regression curve, it is then possible to identify the multiple of the standard deviation of the original loading process for the required fatigue life value (in the case of the required number of kilometres - Fig.9)

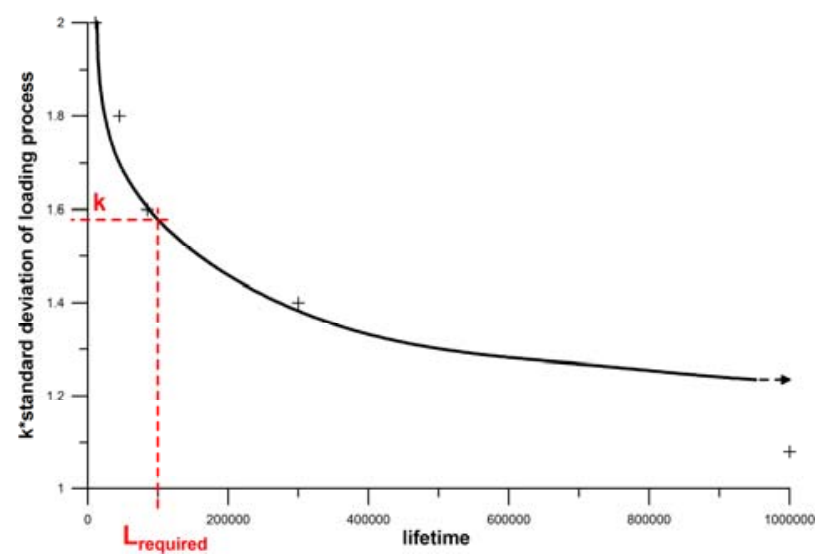

Fig. 9 Finding a multiple $k$ of the standard deviation of the loading process for required fatigue lifetime

Gradual increasing of the peak multiplicity in the loading process, that means its standard deviation, however, leads to the need to correct the peaks obtained on the elasto-plastic state of the material at least from the moment when the slope value is reached. This correction can be performed using a Neuber's hyperbola resulting from an equivalent surface in $\sigma-\varepsilon$ diagram (Fig. 10).

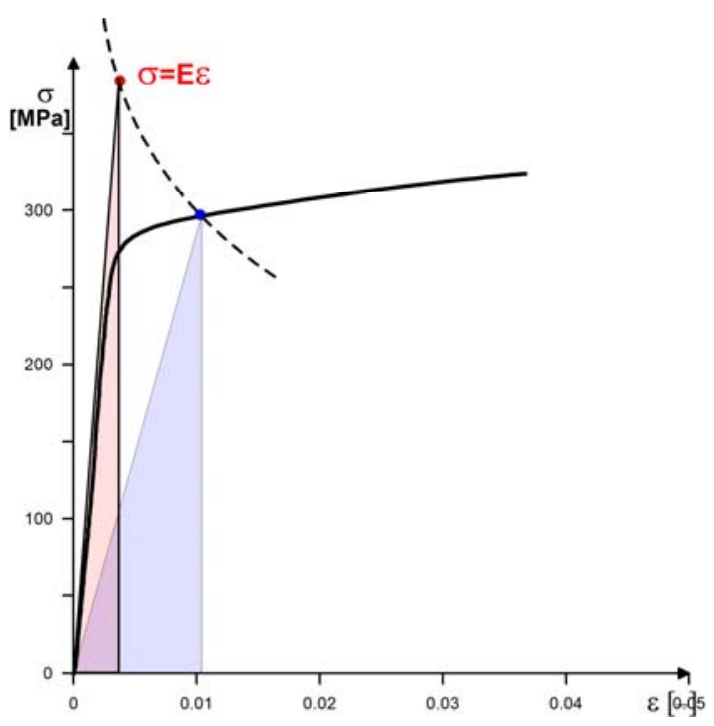

Fig. 10 Neuber method of correcting "linear stresses" on the elastic-plastic state

As material characteristics for elastic-plastic zone can be used cyclic deflection curve, or the static tensile curve. However, after exceeding the "critical" value of the multiplicity of peaks in the loading process, the fatigue lifetime value after the previous reduction begins to increase (Fig. 11).

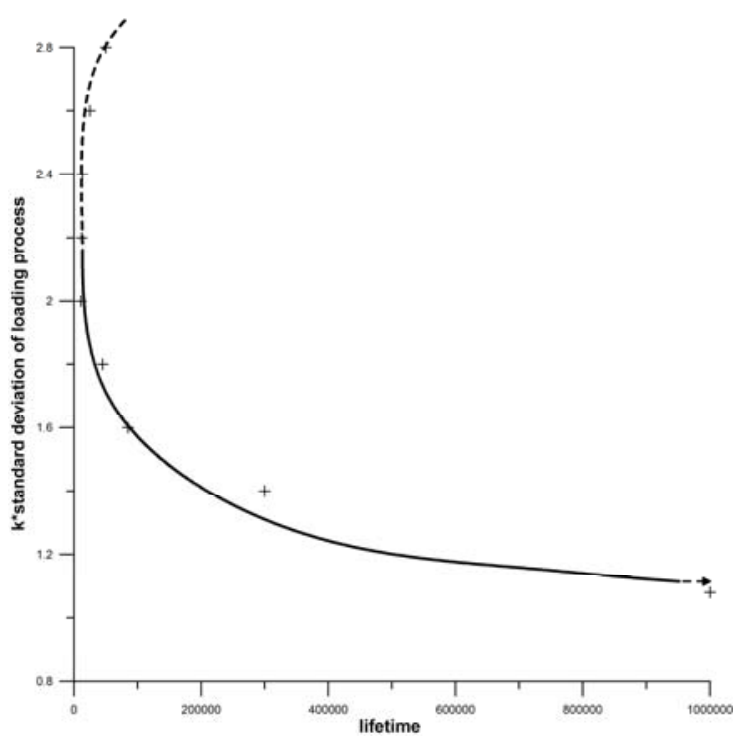

Fig. 11 Development of the standard deviation multiple $\boldsymbol{k}$ of the stress loading process on the fatigue lifetime

This incorrect phenomenon occurs due to the slower increase in peak stresses of peak values in the cycles (corrected stresses in the elastic-plastic state) and the faster increase in lower stresses in cycles (linear multiple). In fact, it starts decreasing the amplitude values in the largest cycles occurs in the overall increase of the mean cycle value. Solving of this disproportion can be the expression of the loading process in the form of amplitudes of strain which, by multiplying their 
peaks, need not to be corrected on the elastic-plastic state.

Using the overall strain process with respect of time for calculation of the fatigue damage requires using of corresponding material properties [4] which can be acquired from the life time curve in the following form

$$
\varepsilon_{a t}=f\left(2 N_{f}\right)
$$

By measuring on specimens created from material of the axle St52 (in the mode of the controlled amplitude of the total strain) is possible to acquire relationship displayed in (Fig. 12).

For the purpose simplification the calculation of the fatigue damage is in this case approximated the life time curve by the following expression

$$
\varepsilon_{a t}=\varepsilon_{d}^{,}\left(2 N_{f}\right)^{d}
$$

This entitles us to a high coefficient of correlation in the regression based on the relationship (Eq. 3) (see Fig. 12).

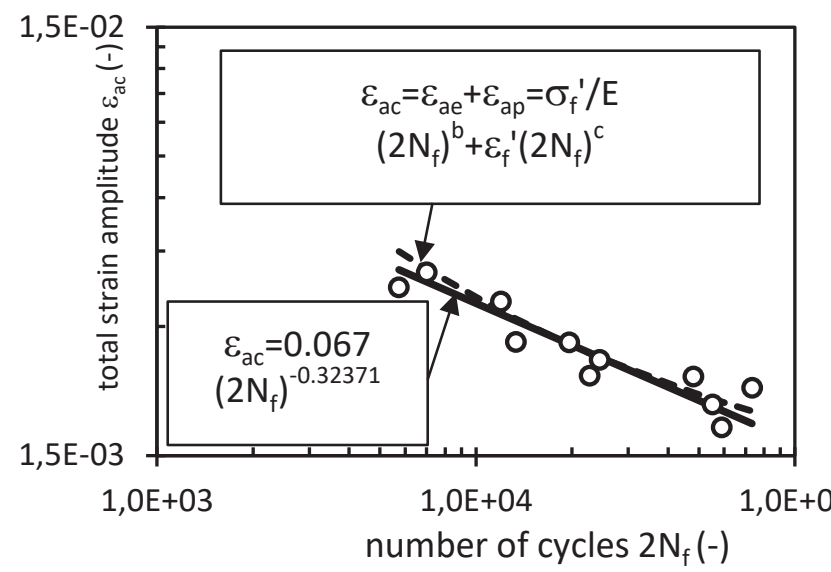

Fig.12. Comparison of the fatigue lifetime curve with experimental results. (o - experimental points)

\section{Conclusions}

The paper presented the analysis of fatigue lifetime and safety against fatigue fracture in the case of caravan axle. For loading processes at the most loaded cross-section point obtained by direct measurement in the form of stresses, the calculated fatigue lifetime values for corresponding pre-fracture risk was calculated with considering the variance of the material parameters. To determine the degree of stress fatigue safety, a multiple of the standard deviation of the process (implemented as a multiple of the peak of the process) was used. For created disproportion of increasing the fatigue lifetime by necessary correction of stress peak values on elasticplastic state, a new approach of assessment has been proposed. This lies in expressing the loading process in the form of the time-depending process of the overall strain amplitude. Then the curve of the dependence of the overall strain amplitude on the number of cycles to the fracture is used for the calculation of the fatigue lifetime. The desired loading multiple by respected requirement for required fatigue lifetime in the form of multiples of the overall strain process can be shown analogous to Fig. 9. In the case of the zero-mean value of the individual loading amplitudes, this multiple of the standard deviation of the process peaks leads to determining the safety of the operation as a direct multiple of the loading amplitudes.

The presented contribution has been prepared under project LO1502 "Development of the Regional Technological Institute" under the auspices of the National Sustainability Program of the Ministry of Education of the Czech Republic aimed to support research, experimental development and.

\section{References}

1. V. Kliman,: Prediction of the random load fatigue life distribution. Fatigue Design, ESIS 16, Edited by J. Solin et al, 1993, Mechanical Engineering Publications, London, p.241-224

2. M. Kepka, M. Kepka, jr., Parametric calculations of fatigue life of critical part of trolleybus rear axle. Procedia Engineering. 213, (2018), s. 227-238.

3. V. Chmelko, V. Kliman, M. Garan, In-time monitoring of fatigue damage. In Procedia Engineering. 101, (2015), p. 93-100

4. V. Chmelko, M. Garan, Long-term monitoring of strains in a real operation of structures. proceedings of the 14th IMEKO TC10 Workshop, Milano (2016), p. 333-336

5. V. Kliman, Fatigue Life Estimation under Random Loading Using the Energy Criterion. Int. J. Fatigue, 7, no. 1 (1985), p.39-44 